\title{
TOPOGRAPHIC AND ENERGETIC HETEROGENEITY STUDIES OF OXIDIZED GRAPHITE SURFACE BY SCANNING TUNNELING MICROSCOPY/SPECTROSCOPY AND PHOTOELECTRON SPECTROSCOPY
}

\author{
Z. KLUSEK \\ Department of Solid State Physics, University of Łódź \\ Pomorska 149/153, 90-236 Łódź, Poland
}

(Received July 9, 1996; revised version October 1, 1996)

\begin{abstract}
Scanning tunneling microscopy and spectroscopy and X-ray photoelectron spectroscopy are used to study oxidation effects of nitric acid on a highly-oriented pyrolytic graphite surface. Various etching times at constant temperature are applied in order to create local binding sites on the surface without creating deep defects. A single and paired chains structure, different from pure graphite at atomic scale, is shown by scanning tunneling microscopy. This can be explained by the presence of oxygenate groups on the surface, revealed by $\mathrm{X}$-ray photoelectron spectroscopy. Both scanning tunneling spectroscopy and X-ray photoelectron spectroscopy demonstrate the vanishing of $\pi$ bands characteristic of $s p^{2}$ graphite hybridization. This, in turn, can be explained by dehybridization related to new bondings of the graphite carbons in the oxygenate groups. An important result of area averaging spectroscopy is the observed energetic heterogeneity considered in terms of the changes of local electronic density of states of the oxidized surface.
\end{abstract}

PACS numbers: 61.16.Ch, 71.20.-b

\section{Introduction}

The creation of binding sites distributed on the basal plane of a layered material such as graphite substrate can be of considerable interest in studies of the distribution of metallic particles on carbon substrate. The first step in this preparation is a chemical oxidation of graphite, which creates active places acting as anchoring sites for metal particles. It is well known that these reactive sites can be obtained by the oxidation treatment of the surface with the use of nitric acid, 
which creates acidic groups. However, most of the previous studies [1-3] show deep defects (pits or holes) as etching effects randomly distributed on the surface. For this reason it is difficult to observe surface modification on the atomic length scale. In early studies [1-5] scanning tunneling microscopy (STM) technique was used to characterize an oxidization process. The studies showed that the oxidization process was not uniform even at small areas.

In this paper the smooth oxidation treatment due to nitric acid at different temperatures and time of etching is proposed, and it is shown that tunneling current can be a direct measure of energetic heterogeneity. In the experiment, the characterization of the oxidized surface in comparison with pure graphite is performed by the combination of three complementary techniques: X-ray photoelectron spectroscopy (XPS), scanning tunneling microscopy, and scanning tunneling spectroscopy (STS). XPS makes it possible to identify precisely the chemical compounds of the surface whereas STM, as a local high-resolution technique, presents images of the atomic modification of the graphite lattice. Scanning tunneling spectroscopy enables us to measure the dependence of tunneling current on the applied voltage, and then to extract detailed information about the local electronic density of states of the sample (LDOS), located around the Fermi level. The aim of the paper is to characterize topographic and energetic heterogeneity created on the surface of highly-oriented pyrolytic graphite (HOPG) by the oxidization treatment. The topographic heterogeneity is considered in terms of the changes of normal corrugation, which is typical of a pure graphite surface. The energetic heterogeneity is considered in terms of the changes of local electronic density of states measured at various places on the oxidized surface. The appearance of such heterogeneity can be interpreted as a non-uniform distribution of oxidized areas on the basal plane and can be described quantitatively. Since the energetic heterogeneity provides information complementary to that obtained by scanning tunneling microscopy, we are able to characterize the surface more effectively by means of both methods.

\section{Experimental section}

A new surface of highly-oriented pyrolytic graphite was prepared by cleavage with adhesive tape before the block of graphite was dipped in the $4 \mathrm{~N}$ solution of nitric acid. This is a standard procedure to create active sites on the graphite surface for catalytic purposes $[1,4,5]$. Different etching times at $70^{\circ} \mathrm{C}$ were used (sample $A-5 \mathrm{~min}$, sample $B-10 \mathrm{~min}$, sample $C-20 \mathrm{~min}$, sample $D-$ $30 \mathrm{~min}$ ). After the acid treatment the samples were rinsed with distilled water and then dried for 30 minutes at $140^{\circ} \mathrm{C}$.

The sample surfaces were analyzed by photoelectron spectroscopy with the use of a W.A. technology spectrometer with conventional monochromatized $\mathrm{Al}_{\alpha}$ source and ultrahigh vacuum conditions. Scanning tunneling microscopy results were obtained by means of either a home-made apparatus with spectroscopy facilities (University of Łódź [6]) or the commercial Besocke Beetle STM with spectroscopy facilities (École Centrale de Lyon). STM investigations were performed in air in the constant current mode, with the typical tunneling current of 1-4 $\mathrm{nA}$. Tips were obtained by electrochemical etching from Pt90-Ir 10 alloy wires. In order to obtain normalized conductance $(\mathrm{d} I / \mathrm{d} V) /(I / V)$ we used the numerical analy- 
sis, but not the phase loop lock technique, to avoid the possible influence of the time constant of a phase detector on the measuring spectra. The microscope was operated in the constant current mode by using digital feedback loop. At the chosen point in the STM $x-y$ scan the digital feedback loop was stopped and within a limited time interval $(\approx 0.04 \mathrm{~s}$ for both polarities) the $I / V$ characteristic was recorded for 128 discrete voltages. The measurements of the current value were made ten times at every bias and then averaged. The results presented were obtained by averaging 4900 individual $I / V$ curves obtained over the $7 \mathrm{~nm} \times 7 \mathrm{~nm}$ area. The $I / V$ curves obtained in the way described above were stored in a laboratory computer and their voltage derivative was obtained. Tunneling spectroscopy data were recorded in the two ranges: from $-4 \mathrm{~V}$ to $+4 \mathrm{~V}$, and from $-1 \mathrm{~V}$ to $+1 \mathrm{~V}$, then the method proposed by Feenstra et al. $[7,8]$ was applied to normalize all the measurements.

\section{Results and discussion}

\subsection{X-ray photoelectron spectroscopy}

Figure 1 shows typical XPS spectra obtained for a graphite sample after cleavage (Fig. 1a) and after oxidization with nitric acid (Fig. 1b) [9]. The graphite surface is inert and no traces of oxygen contamination are observed by XPS. On the oxidized surface the $\mathrm{O} 1 s$ core level is clearly visible. Moreover, at $20-30 \mathrm{eV}$ we can see the satellite on the high energy side of the $\mathrm{C} 1 \mathrm{~s}$ line (Fig. 1a), which is strongly attenuated after oxidation (Fig. 1b). The satellite is typical of plasmon energy loss and its intensity decrease is the first indication that the valence band electrons involved in plasmon excitations have a different configuration after oxidization, in the thin surface layer measured by XPS.

The comparison of $\mathrm{C} 1 s$ photoelectron lines of graphite before and after oxidation is shown in Fig. 2. Additional peak is clearly visible on the oxidized

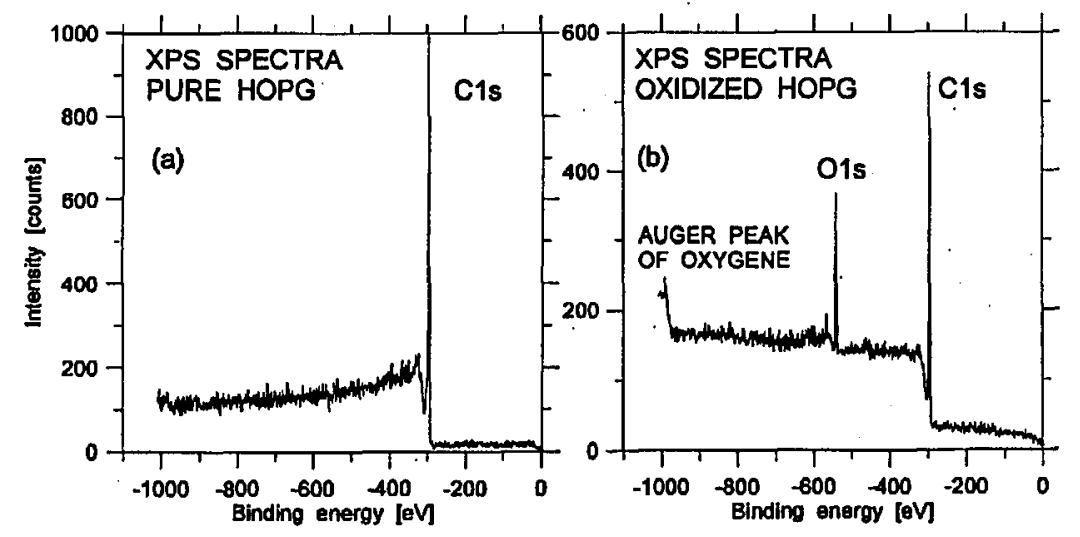

Fig. 1. XPS spectra obtained from a freshly cleaved HOPG surface before (a) and after (b) oxidation in $4 \mathrm{~N} \mathrm{HNO}_{3}$. 


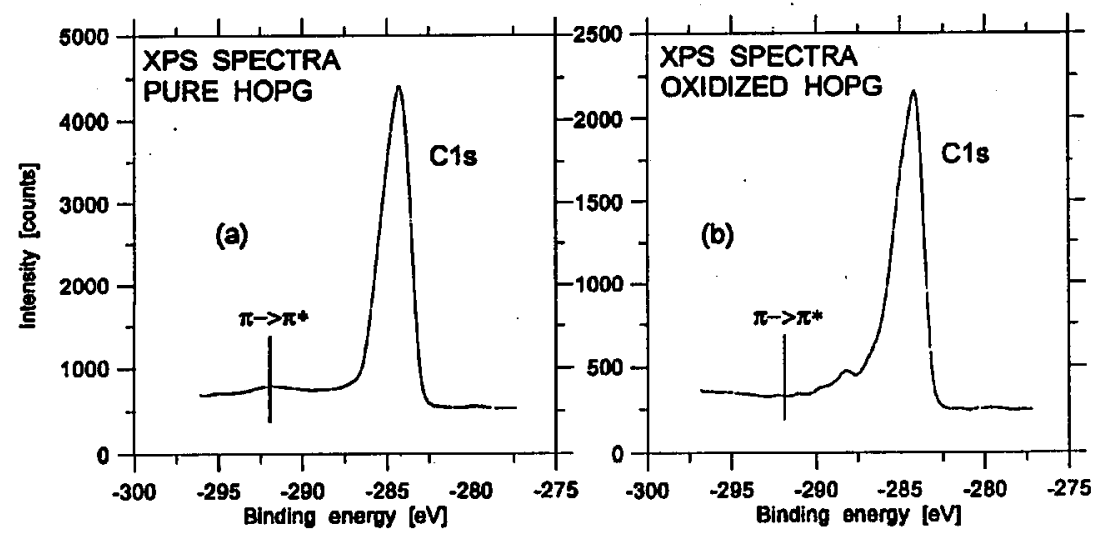

Fig. 2. XPS C $1 s$ peaks from pure (a) and oxidized (b) HOPG surfaces. The peak near $288 \mathrm{eV}$ on (b) is attributed to carboxylic groups. The $\pi \rightarrow \pi$ shake-up satellites are also marked.

sample (Fig. 2b). This peak is due to functionality groups created on the graphite surface by oxidization $[4,5]$. We can also notice that the amplitudes on the high binding energy side of the $\mathrm{C} 1 s$ peak are less intense after oxidation process. This is attributed to $\pi \rightarrow \pi^{*}$ shake-up satellites and involves transitions between occupied and unoccupied $\pi$ bands of graphite [5]. Their decrease is also a strong indication that valence and conduction bands have significantly been affected by oxidation. This modification of the surface electronic structure will be confirmed by tunneling spectroscopy experiments.

\subsection{Scanning tunneling microscopy}

Figure 3 shows a typical atomic resolution image of pure graphite taken in the ambient pressure air. In this case the tunneling microscope shows triangular structure with the spacing of about $0.23 \mathrm{~nm}$ between the maxima in the image. This value is close to the lattice constant of HOPG of $0.246 \mathrm{~nm}$. The small difference (8\%) between the maxima in the image and crystallographic value can be caused by thermal property of our piezo-scanner.

The presented triangular structure is due to the existence of two types of carbon atoms in the hexagonal structure. The carbon atoms, defined as type $A$, have a neighbor in the nearest layer in the distance of $0.335 \mathrm{~nm}$, while the carbon atoms of type $B$ do not have a neighbor in the nearest layer. This site asymmetry of graphite introduces a specific symmetry of a wave function at the Fermi surface, close to the $\bar{K}$ point in the Brillouin zone. Consequently, the Bloch waves of graphite with the energy around the Fermi level have a larger amplitude around the $B$ site than around the $A$ site. The type $B$ atoms contribute in a larger extent to tunneling current than the type $A$ atoms, and a tunneling microscope detects only the type $B$ atoms, whereas the type $A$ atoms remain almost invisible [10]. It confirms that STM does not show an atomic structure of the surface in crystallographic sense, but rather an atomic structure of the surface which is strongly affected by the local electronic density of states. Atomic resolution is also obtained 


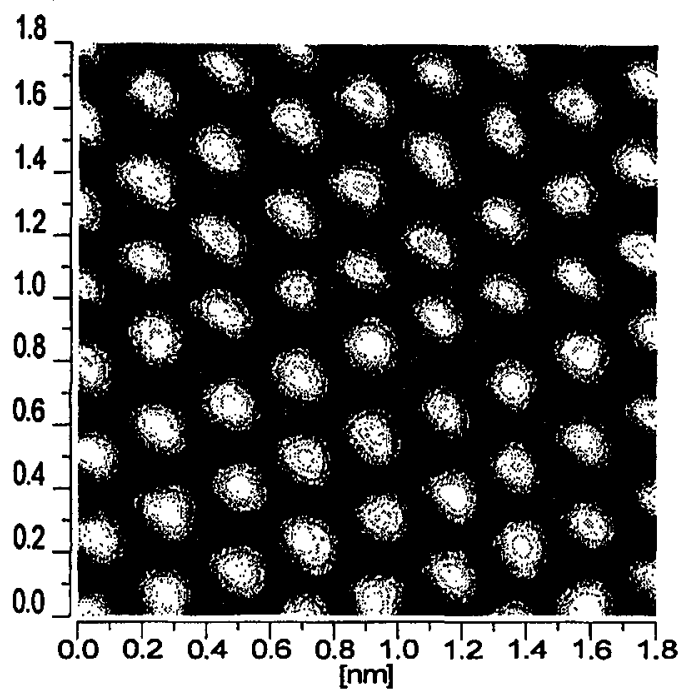

Fig. 3. High resolution STM image of pure graplite. Field of view: $1.8 \mathrm{~nm} \times 1.8 \mathrm{~nm}$, sample biasing: $+100 \mathrm{mV}$, tunneling current: $1 \mathrm{nA}$.

in the case of pure graphite rinsed with distilled water and then dried for $30 \mathrm{~min}$ utes at $140^{\circ} \mathrm{C}$. It leads to the conclusion that this sample treatment does not affect the surface much. Consequently all changes in the case of oxidized graphite should be caused by nitric acid.

STM studies of oxidized samples indicate that the graphite basal plane has undergone an important change. Several samples and several places on sample surfaces have yielded two types of changes.

Firstly, apart from normal corrugation, which is typical of a graphite surface, we also observe the appearance of other structures. In Fig. 4 and Fig. 5 an atomically resolved STM image as well as an atomistic model are presented. The reconstruction appears in the form of chains of bright spots along the [12 $\overline{1} 0] \mathrm{di}-$ rection, with a periodicity of $0.246 \mathrm{~nm}$ corresponding to the interatomic distance along that direction. The distance from $\alpha$ to $\beta$ is $0.738 \mathrm{~nm}$, implying that the structure along the $[2 \overline{1} \overline{1} 0]$ direction repeats itself for every $0.738 \mathrm{~nm}$. The presented image is obtained at the positive sample bias of $100 \mathrm{mV}$ and the tunneling current of $7 \mathrm{nA}$. The images taken at the same sample bias but at different stabilization current values in the range of 2-8 $\mathrm{nA}$ (different tip-sample distances) are similar. The image corrugation depends in a roughly linear fashion on the applied stabilization current. With an increase in current the maxima become more distinct. We suppose that elastic effects between a tip and an oxidized sample might play some role here. Shortly speaking, it is clear that the graphite surface has been modified. The consequence of surface oxidization (registered earlier by X-ray photoelectron spectroscopy) is the creation of oxygenate groups on the surface. To create functional groups, carbon-carbon bondings have to be broken before fixing oxygen or hydroxyl groups. Then the $s p^{2}$ hybridization which describes pure graphite is no longer valid and $s p^{3}$-like bonds form. 


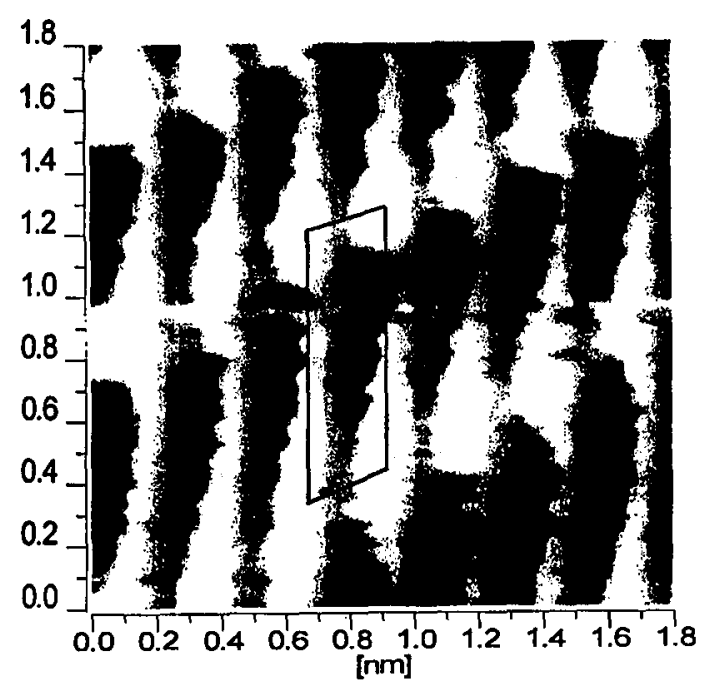

Fig. 4. High resolution STM image of single chains form induced by oxidization on the graphite lattice. Field of view: $1.8 \mathrm{~nm} \times 1.8 \mathrm{~nm}$, sample biasing: $+100 \mathrm{mV}$, tunneling current: $7 \mathrm{nA}$.

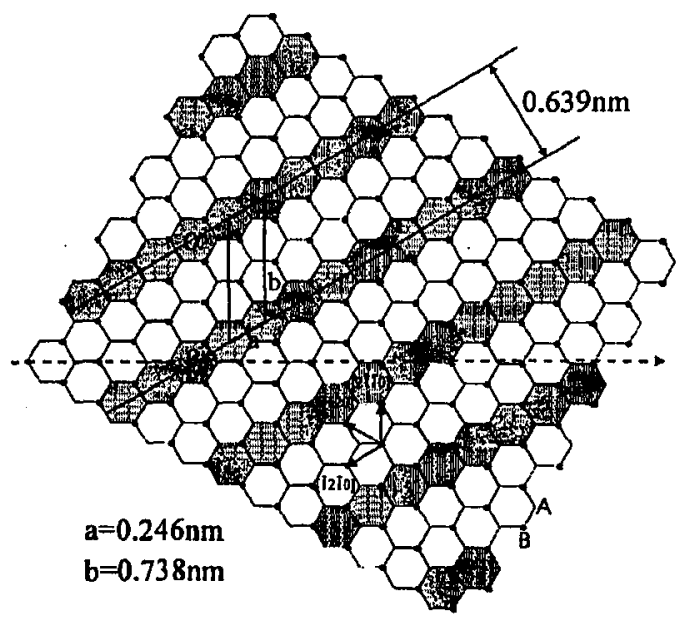

Fig. 5. Atomistic model of the single chains form structure. The small black circles represent the carbon atoms of type $B$, whereas the grey regions represent the oxygenate groups created on the surface.

The other kind of ordered structure differing from a single chain form is observed. Figures 6 and 7 show an atomically resolved STM image as well as an atomistic model of the surface superstructure. The presented image is obtained at the positive sample bias of $100 \mathrm{mV}$ and the tunneling current of $1 \mathrm{nA}$. The superstructure appears in the form of chains of bright spots along the [1120] direction with a periodicity of $0.492 \mathrm{~nm}$ corresponding to the twice interatomic distance 


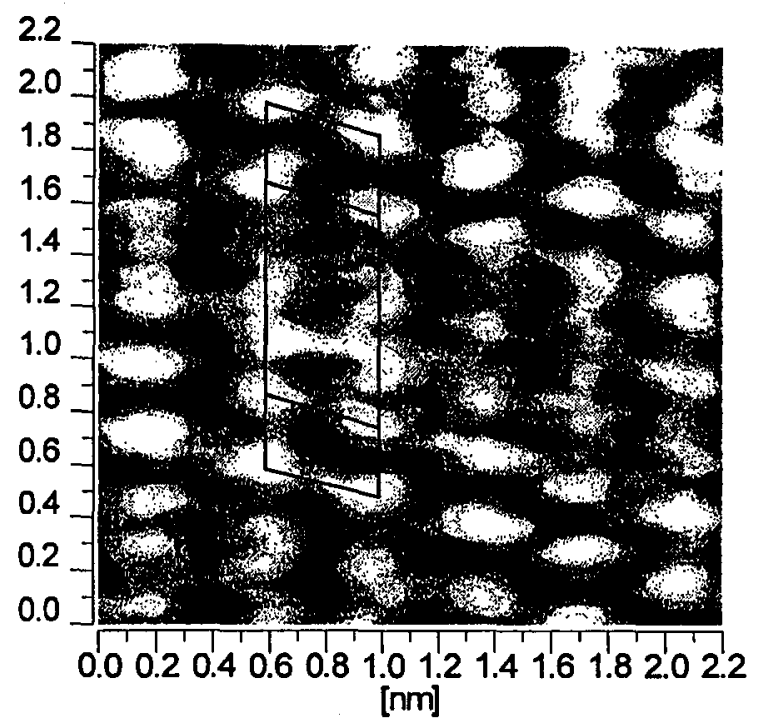

Fig. 6. High resolution STM image of paired chains form induced by oxidization on the graphite lattice. Field of view: $2.2 \mathrm{~nm} \times 2.2 \mathrm{~nm}$, sample biasing: $+100 \mathrm{mV}$, tunneling current: 7 nA.

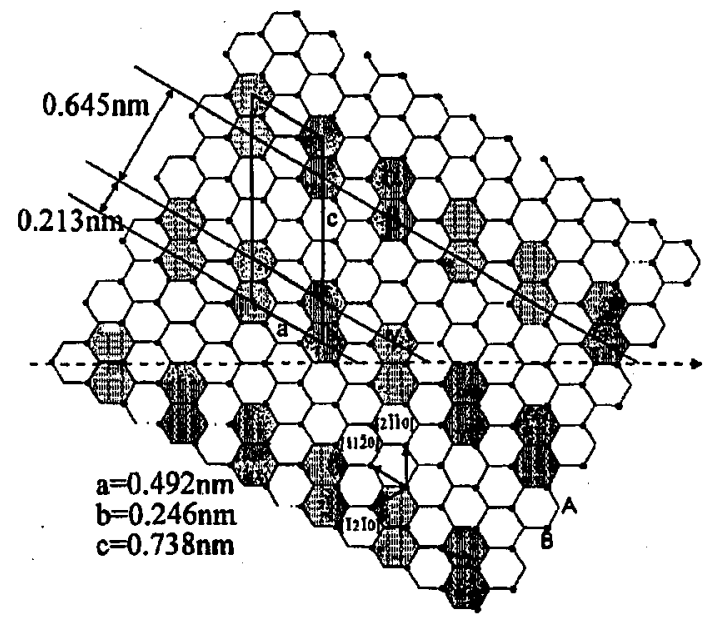

Fig. 7. Atomistic model of the paired chains form structure. The small black circles represent the carbon atoms of type $B$, whereas the grey regions represent the oxygenate groups created on the surface.

along that direction. Furthermore, the chains are paired, and the distance from $\alpha$ to $\beta$ and from $\beta$ to $\gamma$ are $0.246 \mathrm{~nm}$ and $0.738 \mathrm{~nm}$, respectively, implying that structure along the $[2 \overline{1} \overline{1} 0]$ direction repeats itself for every $0.984 \mathrm{~nm}$.

It seems that the obtained images should be interpreted in terms of a fingerprint of the graphite/oxygen electronic interaction rather than as direct images 
of the bonded oxygen atoms. Then, instead of changes in morphology we observe charge density modulations. Moreover, STM images indicate an ordered distribution of functionality groups, which suggests the existence of a short-range ordered interaction instead of a random one. The observed single and paired chains structures are found to be individual phases which coexist together in different areas separated by pure graphite. All the prepared samples show both structures. In many cases we also observe direct transition from one phase to another. The modified regions (where one or both phases can be found) cover small $70-100 \mathrm{~nm}^{2}$ areas surrounded by nearly pure graphite. Different microscopy parameters such as tunneling current (tip-sample distance) and scan speed do not affect the images much. However, the changes of sample bias cause some differences in topography, since different parts of electronic density of states are probed.

Secondly, there are also places on the surface in which tunneling current is quite unstable and defects are in the nanometer scale. These areas should correspond to deeper oxidization of graphite. They are observed first of all in the case of the $C$ and $D$ samples, but it is also seen that the $A$ and $B$ samples, which have originally been less oxidized, have quite similar surfaces after a few days of exposition in the ambient air.

One of the aims of this paper is to modify a graphite surface at the atomic level in order to investigate energetic heterogeneity of the oxidized surface. So far, the best conditions that we could find to create atomic modifications and avoid deep graphite oxidization are those given for the $B$ sample, i.e. 10 min oxidization at $70^{\circ} \mathrm{C}$ in the $4 \mathrm{~N}$ nitric acid. Obviously, it seems difficult to avoid deep oxidization since the defects initially presented on the non-ideal HOPG surface, such as pits and steps, are more favorable sites for oxidization than the graphite basal plane.

\subsection{Scanning tunneling spectroscopy}

The main idea of tunneling spectroscopy experiments is to measure the dependence of tunneling current on the applied voltage $-I / V$. Then it is possible to use tunneling equations to extract a measure of the electronic density of states of a sample $-(\mathrm{d} I / \mathrm{d} V) /(I / V)$.

Figure 8 presents typical current-voltage curves recorded for pure graphite (Fig. 8a) and oxidized graphite (Fig. 8b). We investigate the occupied states below the Fermi level: $-5 \mathrm{~V} \ldots 0 \mathrm{~V}$; the electrons tunnel from the occupied states of the sample to the tip. We also examine the unoccupied states: $0 \mathrm{~V} \ldots+5 \mathrm{~V}$; the electrons tunnel from the tip to the unoccupied states of the sample. Spectra are recorded in a typical manner at the $0.1 \mathrm{~nm}$ intervals over the $7 \mathrm{~nm} \times 7 \mathrm{~nm}$ area with the simultaneous measurements of topography. In Fig. 8 each of the curves consists of an average obtained from 490 spectra which have been acquired individually and averaged in order to improve the signal-to-noise ratio. It is immediately seen that the shape of the curves for pure and oxidized graphite differ, particularly at positive tip bias. Different recordings on oxidized graphite indicate also local differences on that surface after oxidization.

Figure 9 presents the spectroscopic data for pure graphite of the form $(\mathrm{d} I / \mathrm{d} V) /(I / V)$ versus the sample bias which is expected to be related to the 


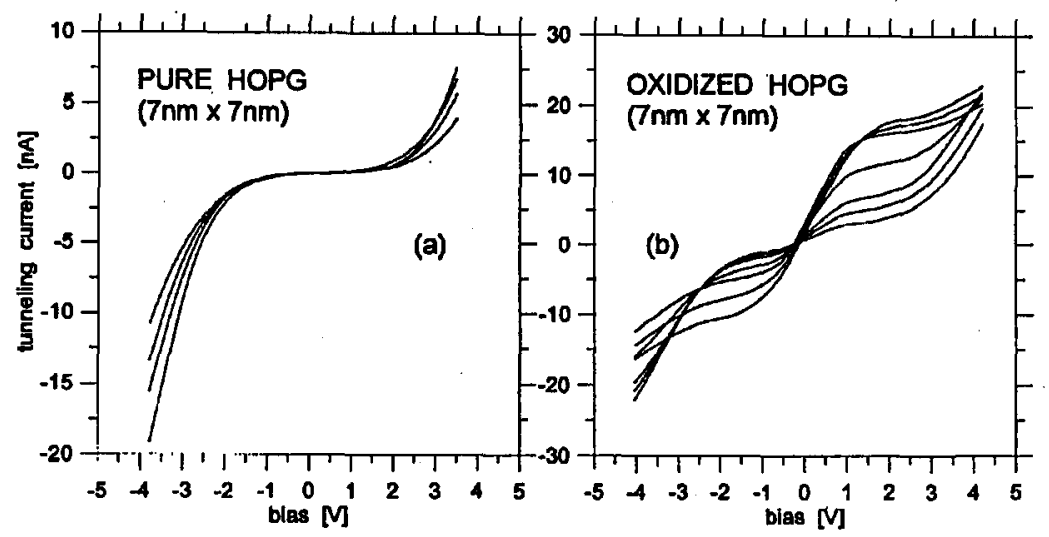

Fig. 8. Different current-voltage curves obtained in different places on pure HOPG (a) and oxidized HOPG (b). Each curve is an average of 490 individual curves obtained from $7 \mathrm{~nm} \times 7 \mathrm{~nm}$ area.

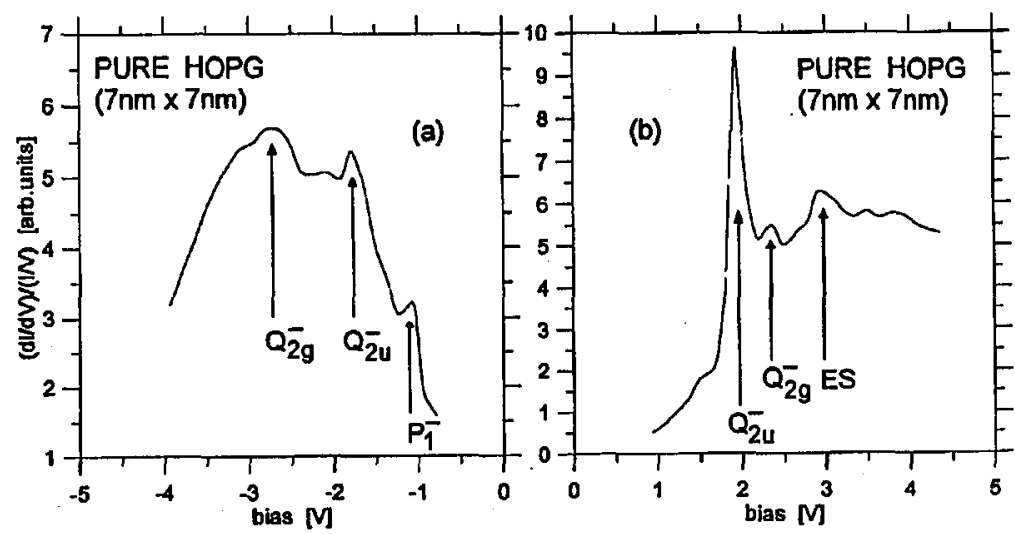

Fig. 9. Normalized tunneling spectroscopy data for occupied (a) and unoccupied (b) electronic states on pure HOPG surface. The curve is an a verage of 490 individual curves obtained from $7 \mathrm{~nm} \times 7 \mathrm{~nm}$ area.

local density of states $[7,8]$. The curve is an average obtained from 490 elementary curves measured over the $7 \mathrm{~nm} \times 7 \mathrm{~nm}$ area.

At the unoccupied spectrum (Fig. 9b), the characteristic features for a pure graphite surface can be distinguished at around $1.7 \mathrm{eV}$ and $2.2 \mathrm{eV}$ and are attributed to the specific points of the Brillouin zone $Q_{2 \mathrm{u}}^{-}$and $Q_{2 \mathrm{~g}}^{-}$, respectively (graphite band structure after Painter and Ellis [11]; symmetry designations based on the modified version by Willis and Fitton [12]). Another structure at $2.7-3 \mathrm{eV}$, denoted as ES, is associated with the extrinsic surface state [10]. On the whole, these results are in good agreement with the experimental investigations by Reihl et al. [13] and by Fusch and Tosatti [14], and with the theoretical researches by Selloni et al. [15]. 
At area-to-area measurements the $(\mathrm{d} I / \mathrm{d} V) /(I / V)$ spectra corresponding to the occupied states of pure graphite (Fig. 9a) show a greater variability than the spectra recorded at the unoccupied states. For the first time the splitting of the $\pi$ valence bands in graphite for the states at $P$ and $Q$ points of the Brillouin zone was observed experimentally by Feuerbacher and Fitton. They used photoemission (PE) spectroscopy, secondary electron emission (SEE) spectroscopy $[16,17]$, and thermoreflectance technique [18]. They observed the state at $0.8 \mathrm{eV}$ below the Fermi level, attributed to the point $P_{1}^{-}$in the Brillouin zone, and two states at $1.8 \mathrm{eV}$ and $2.6 \mathrm{eV}$ below the Fermi level, assigned to the valence states at the $Q_{2 \mathrm{u}}^{-}$ and $Q_{2 \mathrm{~g}}^{-}$points. In our experiments the main feature appears to be a maximum in the electron density of states lying at 2-3 eV (Fig. 9a). It is attributed to a flat band near the $Q_{2 \mathrm{~g}}^{-}$point in the Brillouin zone [19]. A less distinct peak appears at around $1.7 \mathrm{eV}$, which can be interpreted as the $Q_{2 \mathrm{u}}^{-}$state in the Brillouin zone. The last structure observed on this spectrum is located at around $1.1 \mathrm{eV}$ and is tentatively assumed to be the $P_{1}^{-}$state. Although numerous investigations concerning tunneling spectroscopy for HOPG have been performed, not sufficient experimental data for occupied states have been presented. That is why we cannot compare our STS results with other statistics.

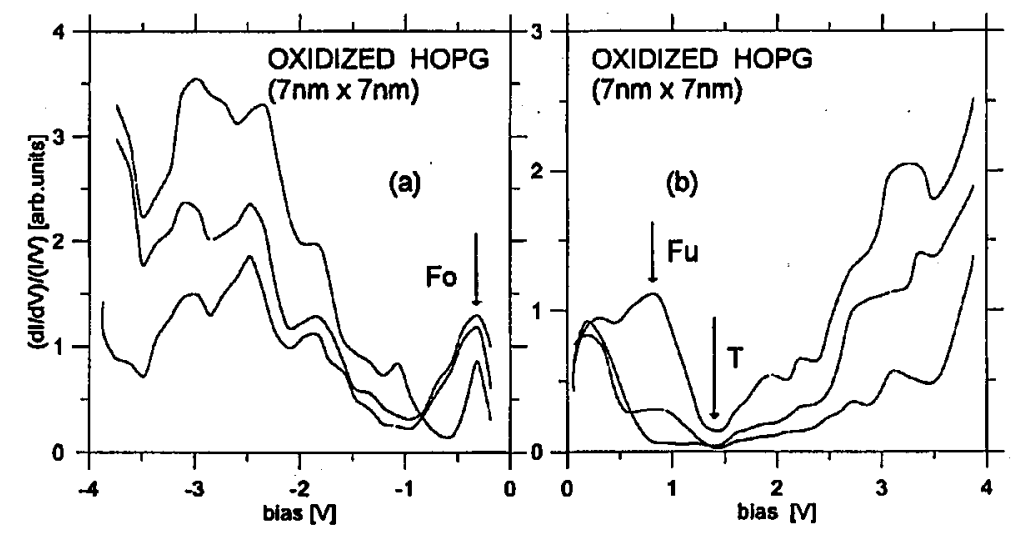

Fig. 10. Normalized tunneling spectroscopy data for occupied (a) and unoccupied (b) states on oxidized HOPG surface. The curve is an average of 490 individual curves obtained from $7 \mathrm{~nm} \times 7 \mathrm{~nm}$ area.

Figure 10b presents four spectroscopic $(\mathrm{d} I / \mathrm{d} V) /(I / V)$ curves corresponding to the unoccupied states for oxidized graphite, each averaged over the $7 \mathrm{~nm} \times 7 \mathrm{~nm}$ area. It is well seen that the spectroscopic data obtained from the oxidized surface differ considerably from the data given by the pure IIOPG surface. The main changes are the almost complete vanishing of the structure at 1-2 eV (denoted as $T$ ), i.e. the disappearance of bulk antibonding unoccupied states of the $\pi$ character mainly, and the relative emergence of the peak at $3-4 \mathrm{eV}$ attributed to the interlayer state [20-22]. We can also observe surface regions in which neither the bulk antibonding unoccupied states nor surface states are detected. In all the cases men- 
tioned an important feature of tunneling spectra is the appearance of the maxima of electron local density of states near the Fermi level, denoted as Fu.

In Fig. 10a the occupied spectra give an evidence of the three types of changes. Firstly, we observe that the peaks are narrower than the peaks in pure graphite and their amplitude is lower. Secondly, there are also places on the surface, where the peaks attributed to the $\pi$ band near the $Q$ point in the Brillouin zone vanish completely. Thirdly, as in the case of unoccupied states, we also observe the appearance of the maximum of electron local density of states (denoted as Fo) near the Fermi level.

It is worth pointing out that tunneling spectroscopy measurements performed along the $[2 \overrightarrow{1} \overrightarrow{1} 0]$ direction with the intervals $0.05 \mathrm{~nm}$ show some correlation with the topography. The shape of the tunneling curves as well as normalized tunneling conductance present the changes with a periodicity of $0.74 \mathrm{~nm}$ and $0.98 \mathrm{~nm}$ for single and paired chains structure, respectively. The tunneling curves performed over the $\alpha, \beta$ and $\gamma$ sites are similar to those presented in Fig. 8b, while tunneling curves taken around these sites resemble the pure graphite spectra. It confirms the well known fact that changes of STM topography are strongly correlated with the local electronic density of states.

A general evolution of the electronic local density of states in the case of oxidized graphite is connected with the vanishing of the $\pi$ bands and the appearance of the maxima of electron local density of states at both sides of the Fermi level. A precise description of these spectroscopic data is not easy but we can notice a coherence between the tunneling spectroscopy and the X-ray photoelectron spectroscopy results. The vanishing of the $\pi$ bands for oxidized graphite is well correlated with the decrease in the $\pi \rightarrow \pi^{*}$ shake-up satellite from the $\mathrm{C} 1 s$ core level. The effect seems less visible for photoelectron spectroscopy since, in terms of a surface itself, it is not as precise as tunneling spectroscopy. Obviously, the consequence of surface oxidization, which has also been demonstrated by X-ray photoelectron spectroscopy, is the creation of oxygenate groups on the surface. In order to create functional groups, carbon-carbon bondings have to be broken before fixing oxygen or hydroxyl groups. Then the $s p^{2}$ hybridization which describes pure graphite is no longer valid and the $s p^{3}$-like bonds form. The $\pi$ bands formed due to the $s p^{2}$ hybridization should disappear when the graphite surface oxidizes, which is clearly seen on $(\mathrm{d} I / \mathrm{d} V) /(I / V)$ or $I / V$ curves.

\subsection{Energetic heterogeneity}

The presented results show that the tunneling spectroscopy can be useful in characterizing the appearance of oxidized places (due to an analysis of the $I / V$ or $(\mathrm{d} I / \mathrm{d} V) /(I / V)$ curves $)$ and their distribution on the HOPG basal plane. On the pure graphite surface we do not observe substantial energetic heterogeneity after the $7 \mathrm{~nm} \times 7 \mathrm{~nm}$ area averaging calculations, considered in terms of changes of the local electronic density of states. It holds when measurements are performed away from the defects and steps on the graphite surface. All the averaged $I / V$ curves measured over different $7 \mathrm{~nm} \times 7 \mathrm{~nm}$ areas are similar, which is presented in Fig. 8a. Besides, the normalized $(\mathrm{d} I / \mathrm{d} V) /(I / V)$ form, derived from the $I / V$ curves, does not show substantial differences. The obtained results confirm a well-known fact 
that a graphite surface is inert, even in the ambient air, which should manifest on the basal plane as homogeneity of the electronic density of states.

On the oxidized surface, in reference to the $7 \mathrm{~nm} \times 7 \mathrm{~nm}$ areas under investigation both $I / V$ (Fig. 8b) and $(\mathrm{d} I / \mathrm{d} V) /(I / V)$ (Fig. 10) measurements indicate differences existing in these areas. During the experiment we have found out that the extent to which a surface has been oxidized is correlated with an increase in the tunneling current at the small bias range $(-1 \mathrm{~V},+1 \mathrm{~V})$. In the case of effective oxidization, instead of a minimal increase in tunneling current (almost flat curve), which is typical of pure HOPG, we observe an abrupt increase in the current. Hence, after oxidization the maxima of the local density of states appear at both sides of the Fermi level (Fig. 10). This effect is accompanied by the vanishing of the $\pi$ bands.

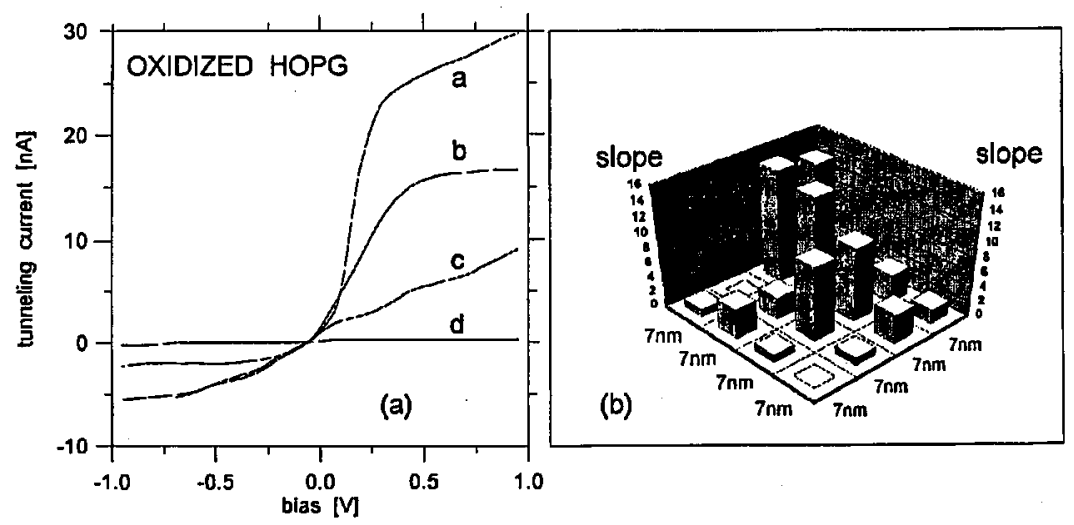

Fig. 11. Typical current-voltage curves recorded for oxidized graphite surface at four different areas $7 \mathrm{~nm} \times 7 \mathrm{~nm}$ close to one another (a). The slope results obtained from sixteen $7 \mathrm{~nm} \times 7 \mathrm{~nm}$ areas for the $B$ sample, 10 min oxidization at $70^{\circ} \mathrm{C}$ in $4 \mathrm{~N}$ nitric acid (b).

In Fig. 11a we present typical current-voltage curves obtained for oxidized graphite surface from four different areas close to one another at the small bias range $(-1 \mathrm{~V},+1 \mathrm{~V})$. The spectroscopic data obtained from these areas differ considerably. The curve denoted as $d$ (Fig. 11a) is very similar to the curves characteristic of pure graphite but only in the bias range of $-1 \mathrm{~V},+1 \mathrm{~V}$ (Fig. 8a). On the other hand, the curve denoted as $a$ (Fig. 11a) in the same range is typical of oxidized graphite (Fig. 8b). In order to quantify a tunneling current behavior we take under consideration the fact that at small biases the tunneling current points are linear. Then, it is possible to fit a line through the points by the use of linear least squares estimation. The line in a two-dimensional space is defined by the equation $I=a+b V$. The slope $b$ is a direct measure of the extent to which the surface has been oxidized and it increases along with an increase in oxidization. In Fig. 11b we present the slope results obtained from sixteen $7 \mathrm{~nm} \times 7 \mathrm{~nm}$ areas for the $B$ sample, i.e. 10 min oxidization at $70^{\circ} \mathrm{C}$ in the $4 \mathrm{~N}$ nitric acid. The slope 
varies from 0 for nearly pure graphite up to 14 for effective oxidization. The finding of energetic heterogeneity on the oxidized surface seems to be an important result of our investigations. It leads to the conclusion that oxidization is not uniform even in very small $7 \mathrm{~nm} \times 7 \mathrm{~nm}$ areas. It is worth noting that the obtained results cannot directly be confirmed by XPS, since this technique gives us only global information whereas tunneling spectroscopy provides us with the local information required. Spectroscopic investigations on several samples and on several places for each sample show that the effective oxidization is confined to very small $50-100 \mathrm{~nm}^{2}$ regions $\left(\right.$ at $70^{\circ} \mathrm{C}, 10 \mathrm{~min}$ ) which are randomly distributed and vary significantly from sample to sample. These regions are surrounded by nearly pure graphite, which is also confirmed by the STM investigations.

It is worth pointing out that the observed reactivity differences show inhomogeneity in electronic structure of graphite due to the surface defects revealed by the studies on the clean surface with the use of small area averaging spectroscopy. It would be interesting to use such type of spectroscopy in the vicinity of the oxidized region in order to see whether it is energetically homogeneous. More research needs to be done to understand the influence of local surface effects on oxidization process.

\section{Conclusions}

Pure and oxidized graphite surfaces were studied by using X-ray photoelectron spectroscopy, and scanning tunneling microscopy and spectroscopy. The XPS data demonstrate the effectiveness of the oxidization process and show the creation of oxygenated surface groups on the basal plane of HOPG. The decrease in a typical $\pi \rightarrow \pi^{*}$ shake-up transition on the $\mathrm{C} 1 s$ photo-peak indicates that valence bands were modified. The STM images for oxidized graphite reveal different atomic structures than for pure HOPG. They prove that local atomic modifications were produced on the basal plane. The tunneling spectroscopy data confirm the fact that the $\pi$ bands vanish after oxidization due to the $s p^{2}$ dehybridization. An important result of our spectroscopic research is finding energetic heterogeneity on the oxidized surface at small areas. The present paper shows how X-ray photoelectron spectroscopy and scanning tunneling microscopy/spectroscopy are complementary to one another in the studies of surface reactivity.

\section{Acknowledgments}

Zbigniew Klusek is grateful to the Ministère des Affaires Étrangères Français for a grant during his stay at the École Centrale de Lyon. Discussions with Prof. L. Porte and Dr. M. Phaner are greatly appreciated.

\section{References}

[1] L. Porte, D. Richard, P. Gallezot, J. Microsc. 152, 515 (1988).

[2] H. Chang, A.J. Bard, J. Am. Chem. Soc. 113, 5588 (1991).

[3] F. Atamny, R. Schlogl, W.J. Wirth, J. Stephan, Ultramiscroscopy 42-44, 660 (1992).

[4] J.S. Hubacek, R.T. Brockenbrough, J. Microsc. 152, 221 (1988).

[5] A. Sartre, M. Phaner, L. Porte, G.N. Sauvion, Appl. Surf. Sci. 70/71, 402 (1993). 
[6] M. Chwialkowski, Z. Klusek, P. Kobierski, W. Olejniczak, M. Slawski, A. Witek, Acta Phys. Pol. A 83, 621 (1993).

[7] R.M. Feenstra, P. Fein, Phys. Rev. B 32, 1394 (1985).

[8] R.M. Feenstra, J.A. Stroscio, A.P. Fein, Surf. Sci. 181, 295 (1987).

[9] Z. Klusek, M. Phaner, P. Krapf, L. Porte, Acta Phys. Pol. A 86, 303 (1994).

[10] R. Wiesendanger, D. Anselmetti, in: Scanning Tunneling Microscopy I, Eds. H.J. Guntherodt, R. Wiesendanger, Springer-Verlag, Berlin 1992, p. 131.

[11] G.S. Painter, D.E. Ellis, Phys. Rev. B 1, 4747 (1970).

[12] R.F. Willis, B.J. Fitton, J. Vac. Sci. Technol. 9, 651 (1972).

[13] B. Reihl, J.K. Gimzewski, J.M. Nicholls, E. Tosatti, Phys. Rev. B 33, 5770 (1986).

[14] H. Fusch, E. Tosatti, Europhys. Lett. 3, 745 (1987).

[15] A. Selloni, P. Carnevali, E. Tosatti, C.D. Chen, Phys. Rev. B 31, 2602 (1985).

[16] B. Feuerbacher, B. Fitton, Phys. Rev. Lelt. 26, 840 (1971).

[17] R.F. Willis, B. Feuerbacher, B. Fitton, Phys. Rev. B 4, 2441 (1971).

[18] M. Anderegg, B. Feuerbacher, B. Fitton, Phys. Rev. Lett. 26, 760 (1971).

[19] F.R. McFeely, S.P. Kowalczyk, L. Ley, R.G. Cavell, R.A. Pollak, D.A. Shirley, Phys. Rev. B 9, 5268 (1974).

[20] M. Posternak, A. Baldereschi, A.J. Freeman, E. Wimmer, M. Weinert, Phys. Rev. Lett. 50, 761 (1983).

[21] Th. Fauster, F.J. Himpsel, J.E. Fischer, E.W. Plummer, Phys. Rev. Lett. 51, 430 (1983).

[22] M. Posternak, A. Baldereschi, A.J. Freeman, E. Wimmer, Pliys. Rev. Lett. 52, 863 (1984). 